\title{
Spontaneous Parasitic Fibroid with Omental Vessel: A Case Report and Literature Review
}

\section{Amala Sunder, MRCPI(OBG) ${ }^{1^{*}}$, Bessy Varghese, MBBS, DGO ${ }^{1}$, Noora Bahzad, MD ${ }^{2}$ and Basma Darwish, $M D^{3}$}

${ }^{1}$ Senior Resident, OBG, Bahrain Defense Force Hospital, Riffa, Bahrain

${ }^{2} J u n i o r$ Resident, OBG, Bahrain Defense Force Hospital, Riffa, Bahrain

${ }^{3}$ Consultant, Department of Obstetrics and Gynecology, Bahrain Defense Force Hospital, Riffa, Bahrain

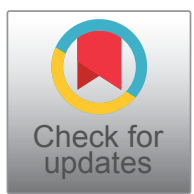

*Corresponding author: Dr. Amala Sunder, MRCPI(OBG), Senior Resident, OBG, Bahrain Defense Force Hospital, Riffa, Bahrain, Tel: 00973-36808905

\begin{abstract}
Aim: This is the case report of a spontaneous parasitic fibroid with omental vessel, and the management at Bahrain Defense Force Hospital.

Case presentation: A 35-years-old female was diagnosed with the pedunculated $20 \mathrm{~cm}$ sub serous fibroid uterus. The pedicle was approximately $2 \mathrm{~cm}$ in length, arising from the fundus of the uterus. A feeding vessel from the omentum was identified, which was providing blood supply to the myoma. She underwent myomectomy after the resection of pedicle and ligation of omental vessels. Post-operative period was uneventful and she had good recovery.

Conclusions: This case report of Parasitic fibroid is a type of subserous fibroid. Generally surgical management is required for the relief of symptoms and to avoid encroachment of nearby abdominal or pelvic structures which in turn leads to further complications.
\end{abstract}

\section{Keywords}

Parasitic fibroid, Omental vessel, Subserous, Feeding vessel

\section{Abbreviations}

CT: Computed Tomography; MRI: Magnetic Resonance Imaging

\section{Background}

Parasitic fibroid is the rare type of subserous uterine fibroid [1,2]. Whenever the subserous fibroid getting attachment to the surrounding structures, they derive the feeding vessel and blood supply from them. Generally they detach from the uterine blood supply and the uterine pedicle often dissipate $[3,4]$. Parasitic fibroids frequently attached to omentum [3]. The other structures of attachment includes urethra, bladder, Gastrointestional tract, intraperitoneal and retroperitoneal myomas [5,6]. Mostly etiology is not well understood $[2,7]$. One of the possibility is post laparoscopic removal of uterine fibroid $[1,8,9]$. Commonly they may not be symptomatic. Mostly diagnosis is incidental. However symptoms are in relation with the size, location, numbers and the attached structure [8]. The common symptoms include abdominal distension, abdominal pain, pelvic pain and pressure symptoms $[2,10]$. Diagnosis could be assisted by imaging such as ultrasonogram, Computed tomography (CT scan) and Magnetic Resonance Imaging (MRI) $[3,8]$. Management is generally surgical ligation of feeding blood vessel and removal of parasitic myoma [10].

\section{Case Presentation}

We report an infrequent case of spontaneous Parasitic Fibroid in a young single female.

She was 35-years-old with the Body Mass Index (BMI) of 23.14. She presented to the Gynecology outpatient clinic, at Bahrain Defense Force Hospital, with the complaints of abdominal distension and

\footnotetext{
Citation: Sunder A, Varghese B, Bahzad N, Darwish B (2021) Spontaneous Parasitic Fibroid with Omental Vessel: A Case Report and Literature Review. Reprod Med Int 4:017. doi.org/10.23937/26434555/1710017

Accepted: August 24, 2021: Published: August 26, 2021

Copyright: (C) 2021 Sunder A, et al. This is an open-access article distributed under the terms of the Creative Commons Attribution License, which permits unrestricted use, distribution, and reproduction in any medium, provided the original author and source are credited.
} 
pressure symptoms since two months. She gave the history of pulsations felt at the level of umbilical region. She did not give any history of loss of appetite/loss of weight/urinary, bowel disturbances. Her menstrual cycle was regular and for the previous three months, less menstrual flow with duration of one day. She was not known to have any medical illness, not gone through any surgical procedures either by laparotomy or via laparoscope. No history of allergies. Her family members all fit and well and the family support was good for her.

Examination of Abdomen appeared apalpable mass occupying almost the entire abdomen three $\mathrm{cm}$ above the umbilicus and up to the Right upper quadrant. Ultra sonogram of abdomen revealed multi lobulated subserous Fibroids each measuring nearly 8 to $10 \mathrm{~cm}$ and all together approximately $20 \mathrm{~cm}$. Adnexal tumors, leomyosarcoma and disseminated peritoneal leiomyomatosis, were considered as differential diagnosis. Magnetic Resonance Imaging (MRI) pelvis reported enlarged uterus riddled with very large myomas measuring as a whole mass of almost $20 \times 12$ $\times 10 \mathrm{~cm}$ which are avidly enhancing mostly sub serosal. Junctional zone is poorly defined due to the presence of extensive myomas. The endometrial stripe is not thickened. No pelvic lymphadenopathy. Even though the etiology of parasitic myomas is not well defined, the previous history of surgical removal of myomas could be one of the reasons. Interestingly our case had no such previous history and it was spontaneous and is the rare occurrence.

Patient was counseled for myomectomy. Upon taking informed consent after explaining possible complications such as bleeding, blood transfusion and injury to bladder /bowel /urinary tracts, she was preceded to surgery. Abdomen was opened through the vertical midline incision in view of the huge size of fibroid. Comprehensive abdomino- pelvis survey was

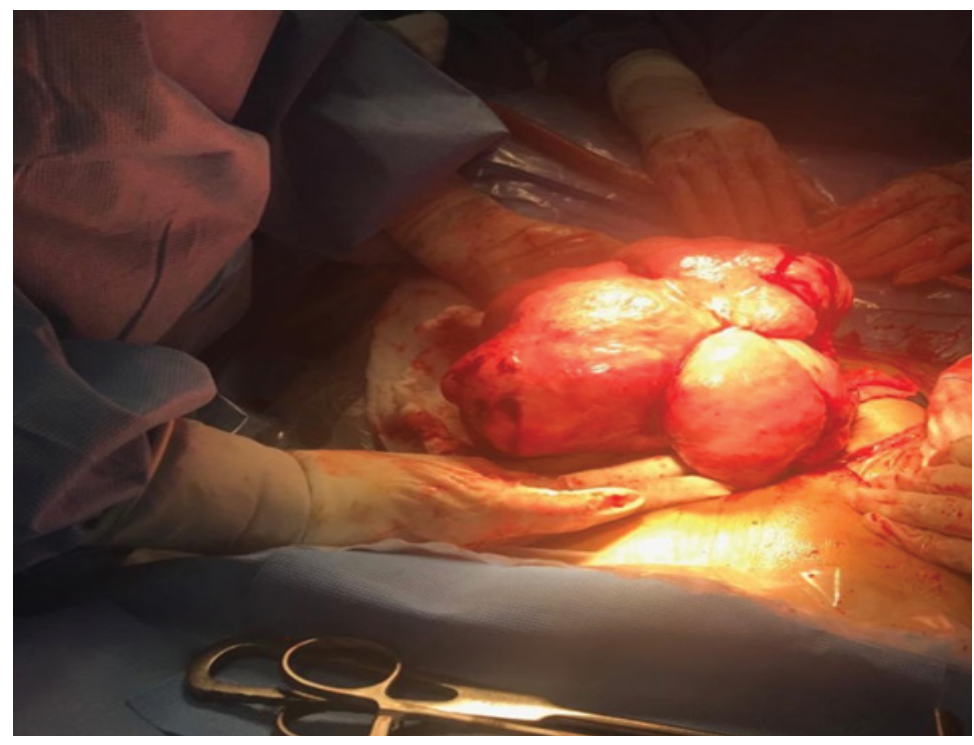

Figure 1: Fibroid mass.
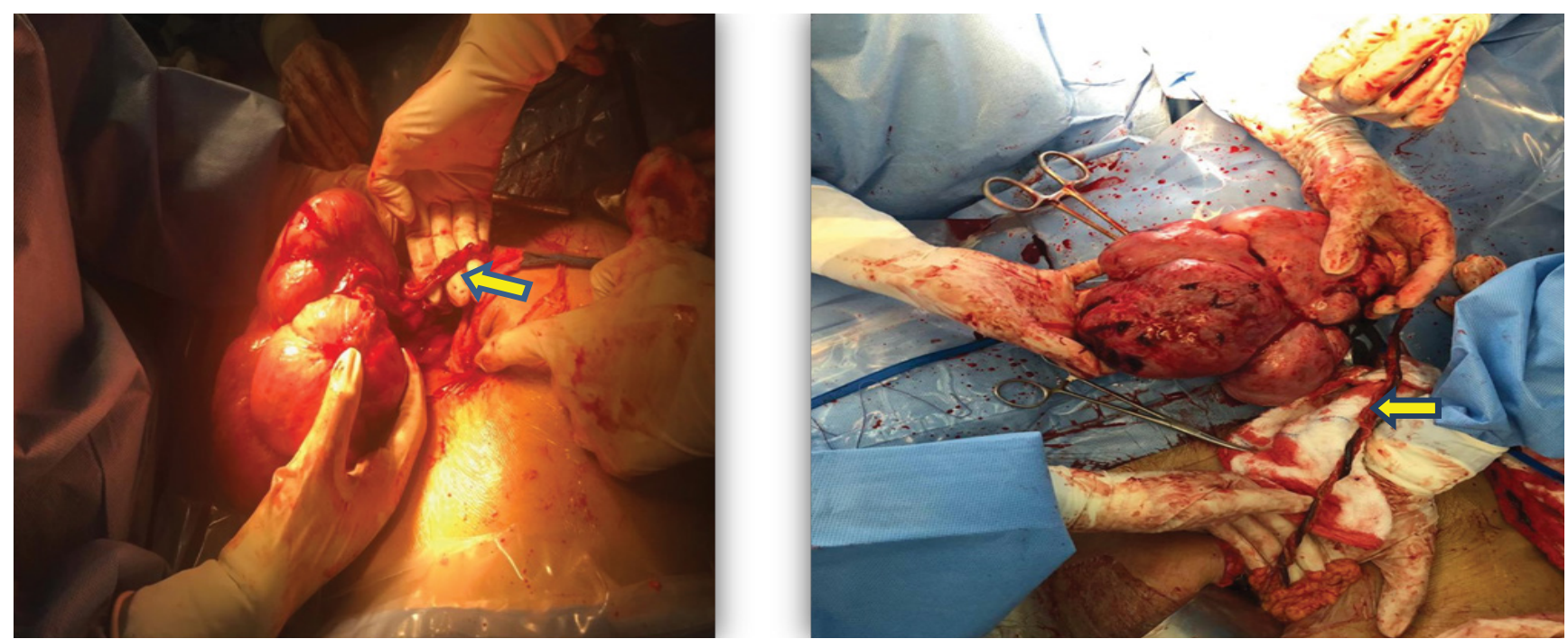

Figure 2 and Figure 3: Fibroid with omental vessel. 
done. Abdominal organs visualized normal. No free fluid. Intraoperatively identified large lobulated mass suggestive of fibroid, attached to the fundus of the uterus with $2 \mathrm{~cm}$ pedicle. Also noticed vessel supply arising from the omentum to the fibroid mass. Bilateral tube and ovary grossly normal. Uterus grossly normal. The Pedicle was identified, which was attached to the fundus of the uterus was clamped and resected. Vascular supply to the fibroid was clamped and ligated (Figure 1, Figure 2 and Figure 3).

Myomectomy was performed successfully. The fibroidmass was extracted and sent for histopathology examination. Hemostasis was secured. In view of huge size Bladder integrity was checked with methylene blue instillation into bladder, and was intact. Abdomen was closed in layers with complete hemostasis. Procedure was uneventful with the Estimated blood loss approximately $500-600 \mathrm{ml}$. Post-operative period was uneventful.

Laboratory Findings: HB 10.6, WBC 7.96, HCT 0.33, PLT 287.BL G A Positive.

She was discharged on $2^{\text {nd }}$ post-operative day without any undue effects.

Histopathology report was uterine fibroid of average cellularity with vessels. No significant mitosis or nuclear atypia seen. Which confirmed the parasitic fibroid with the feeding vessel.

\section{Discussion}

Kelly and Cullen described parasitic leiomyomas as early as 1909 [11]. Their presentation is varied. These unique fibroids were classified by Nezhat and Khointo three categories. The first category spontaneously develops from pedunculated fibroids which detach from the uterus and grow by gaining blood supply from adjacent organs. The second type develops due to reduction in blood flow to the uterus and the third category develops following uterine surgeries [12]. Our case is a spontaneous fibroid in an unmarried lady which is of the second type. The third type of fibroid is suggested to be a complication of morcellator usage in laparascopic surgeries, which occurs due to tissue growth spread in the pelvic cavity concluded Ladke $A B$ and colleagues [13]. As a solution they suggested the usage of endobag morcellation Genetic and hormonal factors may be responsible for parasitic fibroids where a history of morcellation is absent suggested AlTalib A and group [14]. A case report by Alnagar A, et al. revealed a different presentation of the parasitic fibroid where it was attached to the jejunum and the patient presented with small intestinal obstruction [7]. This brings out the complexity in presentation of these unusual leiomyomas.

A similar case to ours was reported by Mushtaq $R$, et al. where a parasitic fibroid along with tortuous omental vessels were identified [15]. Our patient's main concern was the abdominal distension and pressure symptoms. A literature searches on parasitic fibroids by Lete I, et al. gathered information on 274 patients. They found that the mean age was 40 years. $56 \%$ had no uterine surgery and $39 \%$ had a history of morcellation. The clinical symptom frequently encountered was abdominal pain which accounted for $49 \%$ [16]. Our patient was 35-years-old and also did not have any kind of surgery. Ghamande SA and colleagues reported a case with high Ca-125 associated with parasitic leiomyoma [17]. Interestingly, Osegi $\mathrm{N}$ and group also published a case of a parasitic fibroid in a post-menopausal woman who had no history of any previous surgery [4]. Varun $\mathrm{N}$ and colleagues also presented a case of a parasitic fibroid in pregnancy [18]. A case report by Salih AM and group in 2017 concluded that these unique myomas presenting with vague symptoms are diagnosed by ultrasound and resection of the myoma is the management [1]. We used ultrasound and MRI along with clinical assessment for the diagnosis and myomectomy along with the double ligation of the omental vessel as the management.

\section{Conclusions}

Parasitic fibroids have a diverse presentation and causes dilemma when the condition is not kept in mind when dealing with a mass per abdomen. Hence this condition should be considered as a differential diagnosis for early management.

\section{Declarations}

\section{Ethical approval}

The study was approved by the Ethical Committee and Research Centre in Bahrain Defense Force Hospital.

\section{Written consent}

Has been obtained from the patient.

\section{Funding}

None.

\section{Conflicts of Interest}

None.

\section{Authorship}

Amala Sunder: Design, Drafting and writing the manuscript, reviewing the literature and follow up with final approval of the manuscript; Bessy Varghese: Acquisition, literature review and final approval of the manuscript; Noora Bahzad: Literature review and final approval of the manuscript; Basma Darwish: Surgeon Diagnosed the case, performed the operation and follow up. Final approval of the manuscript.

\section{Sponsorship}

None. 


\section{Acknowledgements}

We thank, The Bahrain Defense Force Hospital and The Crown Prince Centre for Training and Medical Research.

\section{References}

1. Salih AM, Kakamad FH, Dahat AH, Habibullah IJ, Rauf GM, et al. (2017) Parasitic leiomyoma: A case report with literature review. Int J Surg Case Rep 41: 33-35.

2. Chrisostomos S, Dimitrios T, Konstantinos Z (2020) Torsion of intestinal parasitic myoma after laparoscopic morcellation: a case report. Journal of Surgical Case Reports 2020: 3.

3. Elagwany AS, Rady HA, Abdeldayem TM (2014) A case of parasitic leiomyoma with serpentine omental blood vessels: An unusual variant of uterine leiomyoma. Journal of Taibah University Medical Sciences 9: 338-340.

4. Osegi N, Oku EY, Uwaezuoke CS, Alawode KT, Afolabi SA (2019) Huge primary parasitic leiomyoma in a postmenopausal lady: A rare presentation. Case reports in obstetrics and gynecology 2019.

5. Putran J, Khaled K (2010) Parasitic leiomyomas: Two case reports and review of literature. Gynecol Surg 7: 383-384.

6. Kho KA, Nezhat C (2009) Parasitic myomas. Obstet Gynecol 114: 611-615.

7. Alnagar A, Essmat A, Eltaweel N, Abdelbaqy TN, Ghazy RM, et al. (2021) Can parasitic fibroid present by intestinal obstruction? Case Report. SN Comprehensive Clinical Medicine 3: 751-753.

8. Oindi FM, Mutiso SK, Obura T (2018) Port site parasitic leiomyoma after laparoscopic myomectomy: A case report and review of the literature. J Med Case Reports 12: 339.
9. Grover A, Bhalla S (2015) Parasitic leiomyoma: A rare complication following laparoscopic myomectomy with review of literature. Curr Med Res Pract 5: 278-281.

10. Khan A, Shawl A, Leung PS (2018) Parasitic leiomyoma of the greater omentum presenting as small bowel obstruction. J Surg Case Rep 2018: 7.

11. Kelly HA, Cullen TS (1909) Myomata of the uterus. Saunders.

12. Nezhat C, Kho K (2010) latrogenic myomas: new class of myomas? Journal of Minimally Invasive Gynecology Sep 17: 544-550.

13. Ladke AB, Palaskar PA, Bhivsane VR (2020) Parasitic Fibroid: Complication of post-laparoscopic morcellation. J Obstet Gynaecol India 71: 207-209.

14. Al-Talib A, Tulandi T (2010) Pathophysiology and possible iatrogenic cause of leiomyomatosis peritonealis disseminata. Gynecol Obstet Invest 69: 239-244.

15. Mushtaq R, Parveen K, Ambreen S (2017) Conjoint presentation of large parasitic leiomyoma and serpentine omental blood vessels: A distinctive case. Journal of the Society of Obstetrics and Gynaecologists of Pakistan 7: 106-109.

16. Lete I, Gonzalez J, Ugarte L, Barbadillo N, Lapuente O, et al. (2016) Parasitic leiomyomas: a systematic review. Eur J Obstet Gynecol Reprod Biol 203: 250-259.

17. Ghamande SA, Eleonu B, Hamid AM (1996) High levels of CA-125 in a case of a parasitic leiomyoma presenting as an abdominal mass. Gynecologic oncology 61: 297-298.

18. Varun N, Elahi AA, Nigam A, Gupta N (2017) Parasitic fibroid during pregnancy: A diagnostic dilemma (Rare Case Report). Indian Journal of Obstetrics and Gynecology Research 4: 458-459. 